\title{
Ovarian stimulation with GnRH analogues
}

\author{
Ivan S. Montenegro ${ }^{1,2}$, Mariana Faller ${ }^{2}$, Isabel C. A. de Almeida², Eduardo P. Passos ${ }^{1,2}$ \\ ${ }^{1}$ Gynecology and Obstetrics Service of the Hospital de Clínicas de Porto Alegre, Assisted Reproductive Sector, \\ Federal University of Rio Grande do Sul \\ ${ }^{2}$ SEGIR- Serviço de Ecografia, Genética e Reprodução Humana
}

\begin{abstract}
Objective: Analysis and compare data between two induction protocols (long agonist and flexible antagonist) in patients submitted an assisted reproduction technique in Porto Alegre.

Methods: Cross-sectional study comparing the intermediate results with the use of two different ovarian stimulation protocols with gonadotropin-releasing hormone agonist versus antagonist to assisted reproductive techniques. The statistical analysis of the retrieved data (age, body mass index, number of oocytes recovered, number of fertilized oocytes, number of oocytes cleaved, total dose of FSH used and ovarian hyperstimulation syndrome) was performed by Student's t-test for parametric data and analysis of covariance for the dependent variables.

Results: A total of 50 patients, 25 in each group, met the criteria for inclusion in the study between January and March in the year 2010. There was statistically significant difference only in the middle ages between the groups ( $P=$ 0.031 ). There was no statistical difference for the remaining data analyzed (body mass index, number of oocytes recovered, number of fertilized oocytes, number of oocytes cleaved and dose of FSH utilized). There were no cases of ovarian hyperstimulation syndrome.

Conclusion: Both protocols are equal in terms of results. The agonist has advantages about scheduling of the procedure, but it takes too long to start the stimulation and have possibility to start medication in a pregnant patient. Added to this, we have the possibility of getting the ovarian hyperstimulation syndrome as complication. In the antagonist group, is clear the ease-of-use of the medication and the fastest start of stimulation.
\end{abstract}

Keywords: IVF outcome/controlled ovarian stimulation/ $\mathrm{GnRH}$ analogue/GnRH agonist/GnRH antagonist

\section{INTRODUCTION}

The reproductive treatment for infertile couples has evolved rapidly since the birth of Louise Brown, the first baby born from in-vitro fertilization (IVF), performed by Steptoe and Edwards in 1978 (Steptoe \& Edwards, 1978). New treatment techniques, new technologies used in the laboratory and new medications make up an arsenal of options in these cases and have helped in the treatment of these couples. The new medications have brought a considerable increase in the chance of pregnancy at the time controlled ovarian stimulation (COS) allowed an increase in the number of oocytes to be recruited and, consequently, greater number of embryos (Yovich, 2011).

The gonadotropin-releasing hormone (GnRH) analogues (agonist and antagonist), in association with exogenous gonadotropins brought the solution to one of the COS's problems: the early peak of luteinizing hormone (LH), which harmed the oocytes collection around $20 \%$ of the cases (Neveu et al., 1987; Ashkenazi et al., 1989). The GnRH agonists' analogues act occupying the GnRH receptors in the pituitary gland causing desensitization. Initially, this medication releases ever follicle-stimulating hormone (FSH) produced and stored in granules, to then, block the production of the pituitary hormones (FSH and LH) (Magon, 2011). The initial pituitary lock occurs between 3 and 4 weeks after the start of the use of the medication and its effect is long-lasting, and may persist for a few weeks after the discontinuation of use (Magon, 2011). Such use prevents the early release of $\mathrm{LH}$, enabling greater control of the stimulus and it is most used in the middle of the luteal phase of the previous cycle (long protocol) (Madani et al., 2012). The advent of antagonist brought another perspective of handling because its use happens to be held at cos cycle itself. The antagonists act in the pituitary gland through a competitive blocking at $\mathrm{GnRH}$ receptors and its action is dose dependent. It occupies the receptors without stimulating the secretion of gonadotropins and the final effect is not to release of pituitary hormones, especially the $\mathrm{LH}$. The onset of action is immediate and the duration of the effect lasts around 34 hours (Schally, 1999; Al-Inany et al., 2011; Gilliam, 2011).

The most recent studies result that two induction protocols are equivalent in terms of retrieved oocytes, embryos fertilized, pregnancy and birth rates. However, they reveal that the protocol that uses antagonist appears to be safer due to the lower occurrence of ovarian hyperstimulation syndrome (OHSS), and it ensures that the patient is not pregnant when the beginning of the use of medicines. Still, the literature has shown a greater need for gonadotropins use with the agonist protocol, resulting in higher cost for patients in addition to the risk of OHSS. (Del Gadillo et al., 2002; Prapas et al., 2005; Aletebi, 2007; Al-Inany et al., 2011; Bodri et al., 2011; Gilliam, 2011).

In our service routinely has been used the agonist protocol, but the possibility of antagonists usage results in greater ease of handling by the user, involves a single menstrual cycle and dispensation guidance for drug use at the end of the previous cycle. Thus, we use the protocol with antagonists and, in this way, we seek to evaluate the use of the two protocols and analyze their results. Added to this, the fact that there is no study that make this comparison in our population.

\section{MATERIAL AND METHODS}

The study constitutes a cross-sectional cohort comparing the intermediate results in assisted reproduction between the $\mathrm{GnRH}$ agonist long protocol (group 1) and flexible GnRH antagonist protocol (group 2), in patients undergoing IVF and intracytoplasmic sperm injection (ICSI) procedure in the year 2010.

For patients in group 1 were administered GnRH agonist, nafarelin acetate $200 \mu \mathrm{g} /$ day (Synarel ${ }^{\circledR}$; Pfizer, USA), intranasal spray, beginning in the 21st day of the cycle prior to stimulation. The administration of recombinant $\mathrm{FSH}$, follitropin $\beta 150 \mathrm{UI} /$ day ( $\mathrm{rFSH}$, Puregon $\AA$; Organon, The Netherlands), s.c, began on day 3 of the cycle. The dose of $\mathrm{GnRH}$ agonist was decreased on that day to $100 \mu \mathrm{g} /$ day 
Table 1. Patient demographic data for the GnRH-antagonist and GnRH-agonist groups

\begin{tabular}{l|c|c|c|}
\hline $\begin{array}{c}\text { Included Pacients } \\
(\mathbf{n}=\mathbf{5 0})\end{array}$ & $\begin{array}{c}\text { Group 1 } \\
\text { Agonist Long Protocol } \\
(\mathbf{n}=\mathbf{2 5})\end{array}$ & $\begin{array}{c}\text { Group 2 } \\
\text { Antagonist Flexible Protocol } \\
(\mathbf{n}=\mathbf{2 5})\end{array}$ & P十 \\
\hline Age (years) & $33.96 \pm 5.00$ & $36.88 \pm 4.26$ & .031 \\
\hline BMI* (kg/m2) & $23.05 \pm 2.96$ & $24.70 \pm 2.80$ & .722 \\
\hline
\end{tabular}

and continued until and including the day of triggering of final oocyte maturation.

In the group 2, patients started daily rFSH treatment with s.c injections of 150 UI of follitropin $\beta$ (Puregon $\AA$, Organon, The Netherlands), on day 3 of cycle. Daily s.c administration of ganirelix $0.25 \mathrm{mg}$ (Orgalutran ${ }^{\circ}$, Organon, The Netherlands) was initiated when at least one follicle measuring $>14 \mathrm{~mm}$ is the ultrasound scanning. Treatment with rFSH and GnRH antagonist continued daily thereafter, until and including the day of triggering of final oocyte maturation.

The dose of rFSH was fixed in both groups, and, in the 5th day of stimulation, the gonadotrophin was changed to highly purified human menopausal gonadotrophin, menotropin $150 \mathrm{UI} /$ day (HMG, Menopur ${ }^{\circ}$, Ferring, Germany), s.c. As soon as three follicles reached a mean diameter of $\geq 17 \mathrm{~mm}$ on ultrasound scanning, recombinant human chorionic gonadotrophin, choriogonadotropin alfa $250 \mu \mathrm{g}$, (rHCG, Ovidrel $®$, Serono, Switzerland) were administered s.c.

Oocyte retrieval was performed 35-36 h after the hCG injection by transvaginal ultrasound-guided needle aspiration. ICSI was performed only in cases with severe male factor or previous fertilization failure. Ultrasound guidance was used for all embryo transfers, which were performed 2 or 3 days post-oocyte retrieval, depending on the weekly clinical program (i.e. to avoid embryo transfer on Sundays). Luteal phase support with $600 \mathrm{mg}$ of micronized progesterone (Utrogestan $®$. Besins-International S.A., France) was initiated in the same day of oocyte retrieval. Were excluded patients with indication of ICSI of testicular biopsy and included patients who failed in low complexity treatments, like intrauterine insemination (IUI). The data were extracted from electronic medical records, being analyzed: date of birth, date of the procedure (ovarian puncture), COS protocol, body mass index (BMI), number of oocytes retrieved, number of fertilized oocytes, number of oocytes cleaved, units of FSH (UFSH) necessary to proceed COS and occurrence of OHSS.

Whereas a difference of up to 3 oocytes as equal among the groups studied, and a standard deviation of 2.7 and 3.2 respectively and each group according to found in the previous study (Gilliam, 2011) were needed at least 34 fertilization cycles, being 17 in each group. Then, for statistical analysis, we used the Student's t-test, Analysis of Covariance (ANCOVA) or Chi-Square test when appropriate, calculated with the program SPSS 16.0. The work was submitted and approved by the Hospital de Clínicas de Porto Alegre's committee of ethics in Research.

\section{RESULTS}

We selected the first fifty patients' files who underwent do IVF/ICSI procedures in 2010 (between January and March), being 25 in each group. The average age of the Group 1 was 36.88 years, statistically greater than group 2 , which was $33.96(P<0.05)$ (table 1$)$. There was no difference between the averages BMI between the groups. Fixing up by age, with the ANCOVA, the comparison of averages oocytes recovered, fertilized oocytes, embryos cleaved and uFSH necessary to proceed COS (table 2). There were no cases of OHSS in both groups.

\section{DISCUSSION}

As already established in the literature, both protocols are similar (Loutradis et al., 2004; Prapas et al., 2005; Xavier et al., 2005; Aletebi, 2007; Kurzawa et al., 2008; Hosseini et al., 2010; Tehraninejad et al., 2010; Al-Inany et al., 2011; Bodri et al., 2011; Gilliam, 2011; Pu et al., 2011; Haydardedeoglu et al., 2012; Pundir et al., 2012). We found the same results in our study.

As demonstrated in this study, there is no difference in oocytes retrieval between these two protocols in recent meta analysis (Tehraninejad et al., 2010; Bodri et al., 2011; Pu et al., 2011; Casano et al., 2012; Pundir et al., 2012) and randomized controlled trials (Loutradis et al., 2004; Xavier et al., 2005; Kurzawa et al., 2008; Tazegul et al., 2008). Differently, some reports showed a less number of oocyte retrieval in the antagonist protocol (Barmat et al., 2005; Arruda et al., 2013; Orvieto \& Patrizio, 2013). Our results could be discussed in another way when we decide to use

Table 2. Assisted reproduction outcomes

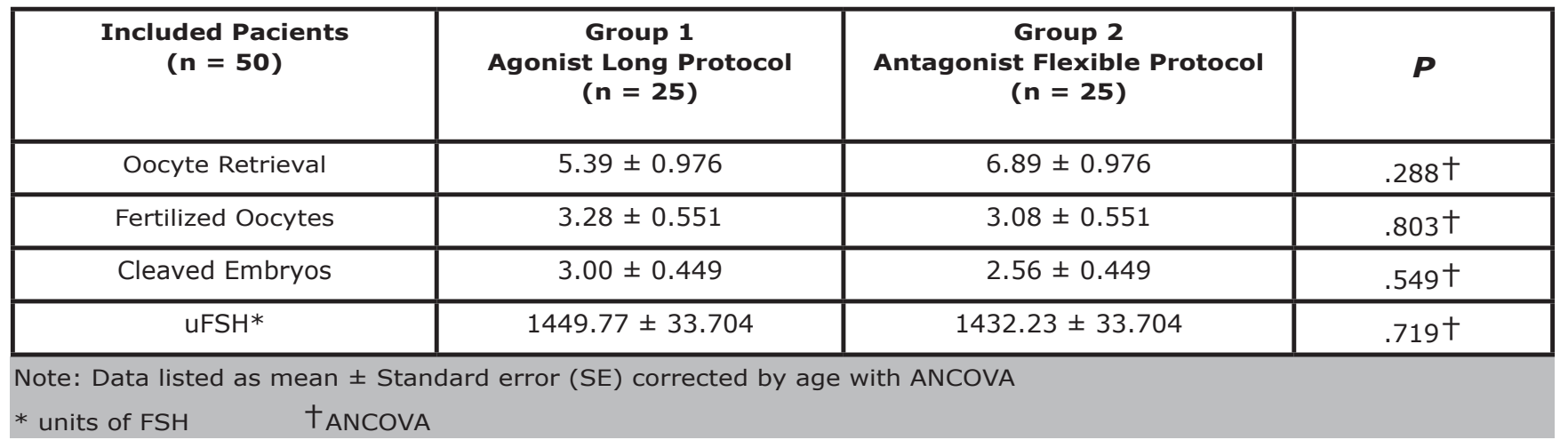


minor dose of gonadotropin. We could say: minor dose, less oocyte, less complication. We are getting more reliable lab results so that we do not want much cryopreserved embryos. In this way we think that a friendly stimulation with a sufficient oocyte to have embryo to be transferred and a little to be cryopreserved should be routine, and, in a future, it should be fertilized only a minimal necessary oocyte and cryopreserve oocyte. Our results demonstrate a more number of oocyte in the antagonist protocol with no significant difference between both groups.

We found no difference in the fertilized oocyte in both groups. We do know the quality of the oocyte is import for the lab results (Xia, 1997) so that in both protocols our operator demonstrates an accuracy of decision in terms of puncture day. Concerning the lab, we could say that we advanced in the last years so that even with some immature we could find good results (Krisher, 2004; Borini \& Bianchi, 2012). It is interesting that all lab results are almost equal in terms of percentage of fertilization and cleavage. So, we could say it depends on the operator experience in the use of those protocols. We do know the more antagonists, the less we get LH so that we should think in a mean LH level in terms of results. Recently, Papanikolaou et al. published a correlation between progesterone levels and pregnancy rates with no difference between protocols but an interesting data in terms of progesterone level, that we got no results because had no routine relating to this data (Papanikolaou et al., 2012).

The antagonist protocol is protective concerning OHSS because even with this probably situation we could trigger with agonist and prevent this important complication (Papanikolaou et al., 2011; Humaidan et al., 2012; Lavorato et al., 2012). This fact is of utmost importance specially if we are leading with younger patients. The major age of the patients was a primary indication for the use of antagonist protocol. Anyway we found this aspect of no importance because our data showed the same number of oocyte collected and no cases of OHSS, probably because our sample was not calculated to find difference in OHSS cases or because we had just one case of polycystic ovary syndrome among the cases studied. We also believe that the minor dose of gonadotropins used in our protocols is responsible for the success in avoid OHSS in both protocols, especially in the agonist protocol.

The agonist is more comfortable for the organization of the oocyte retrieval day but it should be used on the previous cycle and could get patients that might be pregnant (Hayden, 2008). In our study we found no problem about this fact because of the routine imposed but it should be discussed with the group about the facility of some flexible puncture date. Tremellen and Lane showed in an article demonstrating that we could use more antagonist to postpone the puncture day with no adverse results (Tremellen \& Lane, 2010).

After corrected by age, our study shows a minor dose of uFSH in antagonist protocol, but with no significant difference when compared to agonist protocol. Some reports evidence that a less amount of UFSH is needed in antagonist protocol (Kurzawaa et al., 2008; Tazegul et al., 2008; Lainas et al., 2010; Tehraninejad et al., 2010; Pundir et al., 2012; Arruda et al., 2013) and other papers shows no difference between protocols (Barmat et al., 2005; Bodri et al., 2011). The small need of uFSH determine a more friendly stimulation with less risk of OHSS and a greater compliance of our patients in treatment.

\section{CONCLUSIONS}

Finally we should say both protocols are equal in terms of results (Depalo et al., 2012; Luna et al., 2012). The agonist has advantages concerning the mobility but it get to long to start the stimulation and, even, could get a pregnant patient and adjunctive the possibility of getting the OHSS as complication (Zorn et al., 1987; Depalo et al., 2012). In the antagonist group, it is clear the facility of usage and the faster stimulation cycle as it start in the same puncture cycle (Depalo et al., 2012). The idea of impossibility of mobility was not a compliance of our group and should be discussed the possibility of use of more antagonist in case of discomfort of schedule but implicate in a more costs in protocol. It is expected that the availability of evidence-based information on the optimal use of $\mathrm{GnRH}$ antagonists will enhance the probability of pregnancy associated with their application in ovarian stimulation (Xavier et al., 2005). In addition to the flexible antagonist protocol be safer in terms of less likelihood of OHSS and the patient could not be pregnant at the beginning of the cycle (Al-Inany et al., 2011; Depalo et al., 2012), we also consider that the fact of the shorter duration of the stimulus and the least amount of FSH units required are economic factors that also influence the therapeutic decision of the human reproduction team. The smallest amount of gonadotropins directly decreases the costs of treatment, since, in this case, you will need to acquire the least amount of medication to the induction cycle. Already the smallest stimulus duration decreases treatment costs indirectly, as decrease transportation costs to the place of treatment, the shortest time of absence at work and other indirect expenses that are difficult to calculate.

\section{CONFLICT OF INTERESTS}

The authors declare that they have no conflict of interest.

\section{Corresponding author:}

Ivan Sereno Montenegro

Hospital de Clínicas de Porto Alegre

Assisted Reproductive Sector

Rua Ramiro Barcelos, 2350 - Porto Alegre/RS, Brazil

Zip code: 90035903

E-mail: ivan.montenegro@yahoo.com.br

\section{REFERENCES}

Al-Inany H G, Youssef MA, Aboulghar M, Broekmans F, Sterrenburg M, Smit J, Abou-Setta AM. Gonadotrophin-releasing hormone antagonists for assisted reproductive technology. Cochrane Database Syst Rev. 2011: CD001750.

Aletebi F. Comparing gonadotrophin-releasing hormone agonists or gonadotrophin-releasing hormone antagonists in poor responder in IVF. Middle East Fertil Soc J 2007; 12:123-7.

Arruda JT, Approbato MS, Maia MCS, Silva TM, Mendonça C R, Ramos MS. Comparison GnRH agonist short protocol and GnRH antagonist in Brazilian normoresponder patients undergoing their first cycle of controlled ovarian stimulation. JBRA Assist. Reprod. 2013; 17:304-9

Ashkenazi J, Dicker D, Feldberg D, Goldman GA, Yeshaya A, Goldman JA. The value of GnRH analogue therapy in IVF in women with unexplained infertility. Hum Reprod.1989; 4: 667-9.

Barmat LI, Chantilis SJ, Hurst BS, Dickey RP. A randomized prospective trial comparing gonadotropin-releasing hormone (GnRH) antagonist/recombinant follicle-stimulating hormone ( $\mathrm{rFSH}$ ) versus GnRH-agonist/ $\mathrm{rFSH}$ in women pretreated with oral contraceptives be- 
fore in vitro fertilization. Fertil Steril. 2005; 83:321-30.

Bodri D, Sunkara SK, Coomarasamy A. Gonadotropin-releasing hormone agonists versus antagonists for controlled ovarian hyperstimulation in oocyte donors: a systematic review and meta-analysis. Fertil Steril. 2011; 95:164-9.

Borini A, Bianchi V. Oocyte Cryopreservation. In: Seli E, Agarwal A, eds. Fertility Preservation. Emerging Technologies and Clinical Applications. New York: Springer; 2012. p. 89-105.

Casano S, Guidetti D, Patriarca A, Pittatore G, Gennarelli G, Revelli A. MILD ovarian stimulation with GnRH-antagonist vs. long protocol with low dose FSH for non-PCO high responders undergoing IVF: a prospective, randomized study including thawing cycles. J Assist Reprod Genet. 2012; 29:1343-51.

Del Gadillo JC, Siebzehnrübl E, Dittrich R, Wildt L, Lang N. Comparison of GnRH agonists and antagonists in unselected IVF/ICSI patients treated with different controlled ovarian hyperstimulation protocols: a matched study. Eur J Obstet Gynecol Reprod Biol. 2002; 102:179-83.

Depalo R, Jayakrishan K, Garruti G, Totaro I, Panzarino M, Giorgino F, Selvaggi LE. GnRH agonist versus GnRH antagonist in in vitro fertilization and embryo transfer (IVF/ET). Reprod Biol Endocrinol. 2012; 10:26.

Gilliam ML, Gonadotrophin-releasing hormone antagonists for assisted reproductive technology. Obstet Gynecol. 2011; 118:706-7.

Haydardedeoglu B, Kilicdag EB, Parlakgumus AH, Zeyneloglu HB. IVF/ICSI outcomes of the OCP plus GnRH agonist protocol versus the OCP plus GnRH antagonist fixed proto$\mathrm{col}$ in women with PCOS: a randomized trial. Arch Gynecol Obstet. 2012; 286:763-9.

Hayden C. GnRH analogues: applications in assisted reproductive techniques. Eur J Endocrinol, 2008. 159 Suppl $1:$ S17-25.

Hosseini MA, Aleyasin A, Saeedi H, Mahdavi A. Comparison of gonadotropin-releasing hormone agonists and antagonists in assisted reproduction cycles of polycystic ovarian syndrome patients. J Obstet Gynaecol Res. 2010; 36:605-10.

Humaidan P, Papanikolaou EG, Kyrou D, Alsbjerg B, Polyzos NP, Devroey $P$, Fatemi HM. The luteal phase after $\mathrm{GnRH}$-agonist triggering of ovulation: present and future perspectives. Reprod Biomed Online. 2012; 24:134-41.

Krisher RL. The effect of oocyte quality on development. J Anim Sci. 2004; 82 E-Suppl: E14-23.

Kurzawa R, Ciepiela P, Baczkowski T, Safranow K, Brelik P. Comparison of embryological and clinical outcome in $\mathrm{GnRH}$ antagonist vs. GnRH agonist protocols for in vitro fertilization in PCOS non-obese patients. A prospective randomized study. J Assist Reprod Genet. 2008; 25:365-74.

Lainas TG, Sfontouris IA, Zorzovilis IZ, Petsas GK, Lainas GT, Alexopoulou E, Kolibianakis EM.Flexible $\mathrm{GnRH}$ antagonist protocol versus $\mathrm{GnRH}$ agonist long protocol in patients with polycystic ovary syndrome treated for IVF: a prospective randomised con- trolled trial (RCT). Hum Reprod. 2010; 25: 683-9.

Lavorato HL, Petersen CG, Oliveira JBA, Mauri AL, Massaro FC, Cavagna M, Baruffi RLR, Franco Jr JG. [GnRH agonists, GnRH antagonists and Assisted Reproduction]. JBRA Assist. Reprod. 2012; 16: 91-6.

Loutradis D, Stefanidis K, Drakakis P, Milingos S, Antsaklis A, Michalas S. A modified gonadotropin-releasing hormone $(\mathrm{GnRH})$ antagonist protocol failed to increase clinical pregnancy rates in comparison with the long $\mathrm{GnRH}$ protocol. Fertil Steril. 2004; 82:1446-8.

Luna M, Vela G, McDonald CA, Copperman AB. Results with $\mathrm{GnRH}$ antagonist protocols are equivalent to $\mathrm{GnRH}$ agonist protocols in comparable patient populations. J Reprod Med. 2012; 57:123-8.

Madani T, Ashrafi M, Yeganeh LM, Comparison of different stimulation protocols efficacy in poor responders undergoing IVF: a retrospective study. Gynecol Endocrinol. 2012; 28:102-5.

Magon N, Gonadotropin releasing hormone agonists: Expanding vistas. Indian J Endocrinol Metab. 2011; 15:261-7.

Neveu S, Hedon B, Bringer J, Chinchole JM, Arnal F, Humeau C, Cristol P, Viala JL. Ovarian stimulation by a combination of a gonadotropin-releasing hormone agonist and gonadotropins for in vitro fertilization. Fertil Steril. 1987; 47: 639-43.

Orvieto R, Patrizio P. GnRH agonist versus GnRH antagonist in ovarian stimulation: an ongoing debate. Reprod Biomed Online. 2013; 26:4-8.

Papanikolaou EG, Humaidan P, Polyzos N, Kalantaridou S, Kol S, Benadiva C, Tournaye H, Tarlatzis B. New algorithm for OHSS prevention. Reprod Biol Endocrinol. 2011; 9:147.

Papanikolaou EG, Pados G, Grimbizis G, Bili E, Kyriazi L, Polyzos NP, Humaidan P, Tournaye H, Tarlatzis B GnRH-agonist versus $\mathrm{GnRH}$-antagonist IVF cycles: is the reproductive outcome affected by the incidence of progesterone elevation on the day of HCG triggering? A randomized prospective study. Hum Reprod. 2012;27:1822-8.

Prapas N, Prapas Y, Panagiotidis Y, Prapa S, Vanderzwalmen $P$, Schoysman R, Makedos G. GnRH agonist versus GnRH antagonist in oocyte donation cycles: a prospective randomized study. Hum Reprod. 2005; 20:1516-20.

Pu D, Wu J, Liu J, Comparisons of GnRH antagonist versus GnRH agonist protocol in poor ovarian responders undergoing IVF. Hum Reprod. 2011; 26:2742-9.

Pundir J, Sunkara SK, El-Toukhy T, Khalaf Y. Meta-analysis of GnRH antagonist protocols: do they reduce the risk of OHSS in PCOS? Reprod Biomed Online. 2012; 24: 6-22. Schally AV. Luteinizing hormone-releasing hormone analogs: their impact on the control of tumorigenesis. Peptides. 1999; 20:1247-62.

Steptoe PC, Edwards RG. Birth after the reimplantation of a human embryo. Lancet. 1978; 2:366.

Tazegul A, Görkemli H, Ozdemir S, Aktan TM. Comparison of multiple dose GnRH antagonist and minidose long 
agonist protocols in poor responders undergoing in vitro fertilization: a randomized controlled trial. Arch Gynecol Obstet. 2008; 278: 467-72.

Tehraninejad ES, Nasiri R, Rashidi B, Haghollahi F, Ataie M. Comparison of $\mathrm{GnRH}$ antagonist with long $\mathrm{GnRH}$ agonist protocol after OCP pretreatment in PCOs patients. Arch Gynecol Obstet. 2010; 282: 319-25.

Tremellen KP, Lane $\mathrm{M}$. Avoidance of weekend oocyte retrievals during $\mathrm{GnRH}$ antagonist treatment by simple advancement or delay of hCG administration does not adversely affect IVF live birth outcomes. Hum Reprod. 2010; 25: 1219-24.

Xavier P, Gamboa C, Calejo L, Silva J, Stevenson D, Nunes A, Martinez-de-Oliveira J. A randomised study of $\mathrm{GnRH}$ antagonist (cetrorelix) versus agonist (busereline) for controlled ovarian stimulation: effect on safety and efficacy. Eur J Obstet Gynecol Reprod Biol. 2005; 120: 185-9.

Xia P. Intracytoplasmic sperm injection: correlation of oocyte grade based on polar body, perivitelline space and cytoplasmic inclusions with fertilization rate and embryo quality. Hum Reprod. 1997;12:1750-5.

Yovich JL. A clinician's personal view of assisted reproductive technology over 35 years. Reprod Biol. 2011; 11 Suppl 3: $31-42$.

Zorn JR, Boyer P, Guichard A. Never on a Sunday: programming for IVF-ET and GIFT. Lancet. 1987; 1 : 385-6. 